BMJ Open Diabetes Research \& Care

\section{Cord serum metabolome and birth weight in patients with gestational diabetes treated with metformin, insulin, or diet alone}

To cite: Huhtala MS, Rönnemaa T, Pellonperä 0 , et al. Cord serum metabolome and birth weight in patients with gestational diabetes treated with metformin, insulin, or diet alone. BMJ Open Diab Res Care 2021;9:e002022. doi:10.1136/ bmjdrc-2020-002022

- Supplemental material is published online only. To view, please visit the journal online (http://dx.doi.org/10.1136/ bmjdrc-2020-002022).

Received 20 November 2020 Accepted 9 May 2021

Check for updates

(C) Author(s) (or their employer(s)) 2021. Re-use permitted under CC BY-NC. No commercial re-use. See rights and permissions. Published by BMJ.

${ }^{1}$ Obstetrics and Gynecology, University of Turku, Turku, Finland

${ }^{2}$ Obstetrics and Gynecology, TYKS Turku University Hospital, Turku, Finland

${ }^{3}$ Medicine, University of Turku, Turku, Finland

${ }^{4}$ Medicine, TYKS Turku University Hospital, Turku, Finland

Correspondence to Dr Mikael S Huhtala; misahu@utu.fi

\section{ABSTRACT}

Introduction Recent research has demonstrated the benefits of metformin treatment in gestational diabetes (GDM) on short-term pregnancy outcomes (including excessive fetal growth and pre-eclampsia), but its effects on fetal metabolism remain mostly unknown. Our aim was to study the effects of metformin treatment compared with insulin or diet on the cord serum metabolome and also to assess how these metabolites are related to birth weight (BW) in pregnancies complicated by GDM.

Research design and methods Cord serum samples were available from 113, 97, and 98 patients with GDM treated with diet, insulin, and metformin, respectively. A targeted metabolome was measured using nuclear magnetic resonance spectroscopy. The patients in the metformin and insulin groups had participated in a previous randomized trial (NCT01240785).

Results Cord serum alanine was elevated in the metformin group $(0.53 \mathrm{mmol} / \mathrm{L})$ compared with the insulin $(0.45 \mathrm{mmol} / \mathrm{L}, \mathrm{p}<0.001)$ and the diet groups $(0.46 \mathrm{mmol} / \mathrm{L}, \mathrm{p}<0.0001)$. All other measured metabolites were similar between the groups. The triglyceride (TG)to-phosphoglyceride ratio, average very low-density lipoprotein particle diameter, docosahexaenoic acid, omega- 3 fatty acids (FAs), and ratios of omega-3 and monounsaturated $F A$ to total FA were inversely related to BW. The omega-6-to-total-FA and omega-6-to-omega3-FA ratios were positively related to $\mathrm{BW}$. Cholesterol in very large and large high-density lipoprotein (HDL) was positively $(p<0.01)$ associated with BW when adjusted for maternal prepregnancy body mass index, gestational weight gain, glycated hemoglobin, and mode of delivery. Conclusions Metformin treatment in GDM leads to an increase in cord serum alanine. The possible long-term implications of elevated neonatal alanine in this context need to be evaluated in future studies. Although previous studies have shown that metformin increased maternal TG levels, the cord serum TG levels were not affected. Cord serum HDL cholesterol and several FA variables are related to the regulation of fetal growth in GDM. Moreover, these associations seem to be independent of maternal confounding factors.

Trial registration number NCT01240785.

\section{INTRODUCTION}

Gestational diabetes (GDM) is a growing health concern that affects both mothers

\section{Significance of this study}

What is already known about this subject?

- Metformin causes alterations in the maternal metabolome during pregnancy.

- Metformin may be superior to insulin regarding short-term perinatal outcomes. However, the shortterm and long-term metabolic implications for the offspring are not known.

What are the new findings?

- The offspring of metformin-treated mothers had higher cord serum alanine levels compared with the insulin-treated and diet-treated groups.

- Metformin treatment in gestational diabetes does not appear to affect the cord serum lipid profile or cord serum lactate or ketone levels.

How might these results change the focus of research or clinical practice?

- Although metformin causes an elevation in maternal triglycerides, it was reassuring to find that the lipid profile of the neonate as examined using cord serum was not affected.

- The long-term implications of elevated cord serum alanine need to be evaluated in the future.

and neonates worldwide. It increases the risk of complications such as macrosomia, pre-eclampsia, neonatal hypoglycemia and hyperbilirubinemia, and the need for neonatal intensive care. ${ }^{1}$ In the long term, GDM increases especially the risk for type 2 diabetes in mothers ${ }^{2}$ and also metabolic syndrome and obesity in the offspring. ${ }^{3}$ The most common pharmacological treatment options for GDM are insulin and metformin; both affect maternal glucose, lipid, ${ }^{4}$ and amino acid $^{5}$ metabolism.

When diet and lifestyle modifications alone are insufficient to control maternal hyperglycemia, a pharmacological treatment (either metformin or insulin) is initiated. Compared with insulin, metformin appears superior at reducing risk of neonatal hypoglycemia, 
macrosomia, large-for-gestational age (LGA) neonate, pre-eclampsia, and neonatal intensive care unit admission. ${ }^{6}$ However, metformin crosses the placenta $^{7}$; in follow-up studies, metformin exposure in utero has been associated with increased prepubertal anthropometric variables such as weight, Body Mass Index (BMI), and waist circumference in offspring, ${ }^{8} 9$ which has led to concerns regarding metformin's long-term effects on offspring's health. Whether these effects are promoted by alterations in fetal metabolism remains unknown. A previous trial comparing metformin and insulin treatment in GDM revealed no differences in cord serum triglyceride (TG), high-density lipoprotein (HDL) cholesterol, and low-density lipoprotein (LDL) cholesterol levels. ${ }^{10}$ To our knowledge, no previous studies have evaluated the effect of antenatal metformin exposure on the cord serum metabolome (a proxy of fetal metabolism) in detail.

In addition to genetic factors, adequate nutrition, and placental blood flow, ample evidence supports the role of maternal serum metabolites in fetal growth. ${ }^{11}$ Particularly in pregnancies complicated by GDM, maternal serum glucose, ${ }^{1}$ lipids, ${ }^{12}$ and amino acids ${ }^{513}$ have been related to fetal growth. Furthermore, it has been shown that cord serum metabolites are associated with birth weight $(\mathrm{BW})^{1415}$ and may also be associated with the long-term metabolic health of the offspring, although these studies have yielded divergent results. ${ }^{16} 17$

The aim of this study was to evaluate the possible effects of metformin treatment of GDM in the neonate cord serum targeted metabolome compared with maternal treatment with insulin or lifestyle modifications alone. Our second aim was to evaluate the clinical impact of the variation in cord serum metabolome. Due to low overall incidence of adverse perinatal outcomes in the study ${ }^{18}$ and the apparent dysregulation of fetal growth in GDM, BW was chosen as the outcome of interest. Thus, we examined to which extent these metabolites are associated with BW in GDM and whether these associations are modified by GDM treatment.

\section{RESEARCH DESIGN AND METHODS}

The study population treated with antihyperglycemic drugs was derived from a previous randomized trial that compared metformin and insulin in participants with GDM. ${ }^{18}$ In addition, patients diagnosed with GDM but not requiring pharmacological treatment (described in ${ }^{19}$ ) were included in the present study. The study participants were recruited at Turku University Hospital (Finland) between June 2006 and December 2010. The GDM diagnosis was made according to Finnish national criteria based on a 2-hour $75 \mathrm{~g}$ oral glucose tolerance test (OGTT). The diagnostic cut-off values were $\geq 4.8$ (fasting), $\geq 10.0$ ( 1 hour) and $\geq 8.7 \mathrm{mmol} / \mathrm{L}$ (2 hours) until the release of new guidelines in December 2008; thereafter, cut-off values were $\geq 5.3, \geq 10.0$, and $\geq 8.6 \mathrm{mmol} / \mathrm{L}$, respectively. At least two elevated values were required. The patients in the study were diagnosed at a mean of $27.0 \quad(\mathrm{SD}=2.6)$ gestational weeks. Pharmacological treatment was indicated if the patient had recurrent hyperglycemia (fasting glucose $\geq 5.5$ and/or postprandial glucose $\geq 7.8 \mathrm{mmol} / \mathrm{L}$ ) despite diet and lifestyle modifications. The patients who required pharmacological treatment were randomized to either the metformin or insulin groups at a mean of 30 gestational weeks, as described previously. ${ }^{18}$ Metformin was started at a daily dose of $500 \mathrm{mg}$ and increased up to $2000 \mathrm{mg}$ if needed (median: $1500 \mathrm{mg}$ ). For insulin treatment, neutral protamine Hagedorn insulin and/ or rapid-acting insulin (lispro or aspart) were used. The study was registered with ClinicalTrials.gov (http://clinicaltrials.gov/ct2/show/NCT01240785).

Glycated hemoglobin (HbA1c) and fasting serum C-peptide levels were analyzed at the time of the GDM diagnosis using routine laboratory methods. BW was expressed in gram and SD units (the deviation from the Finnish population mean BW adjusted for gestation duration). ${ }^{20}$ Small-for-gestational age (SGA) and LGA neonates were classified using BWs $>90$ th percentile and $<10$ th percentile, respectively.

In the present study, we had cord serum samples available from 113, 97, and 98 neonates in the diet, insulin, and metformin groups, respectively. Twenty-one percent of patients who were randomized to receive metformin required additional insulin. These patients were included in the metformin group in the analyses because we argued that the possible untoward metabolic effects of metformin exposure would also be present in patients receiving both metformin and insulin. Moreover, these patients were older and had higher HbAlc at the time of diagnosis than the rest of the metformin group, and therefore exclusion of these patients would introduce bias. The effect of exclusion was, however, evaluated in a sensitivity analysis. Mixed arterial and venous cord blood was taken immediately after delivery from the placental side of the cut umbilical cord by letting the blood flow freely into test tubes. The samples were allowed to clot at room temperature and then were centrifuged at $3000 \mathrm{rpm}$ for $5 \mathrm{~min}$ to obtain serum, which was stored at $-70^{\circ} \mathrm{C}$ prior to further analyses.

A targeted metabolic biomarker profile that included lipids, lipoproteins, amino acids, glycolysis-related metabolites, and ketones (142 metabolic measures in total, online supplemental table 1) was analyzed using highthroughput proton nuclear magnetic resonance (NMR) spectroscopy (Nightingale Health, Helsinki, Finland). ${ }^{21}$ The sample preparation, NMR spectra acquisition, metabolite identification, and quantification process have been described in detail in the online supplemental file 1). Due to the rejection of individual measurements by quality control, there was a slight difference in the number of successfully quantified samples for various metabolic measures. The NMR protocol has been widely applied in epidemiological research, and the quantification accuracy has shown high consistency when compared with routine clinical chemistry methods (eg, 
the correlation coefficients between NMR and routine clinical chemistry methods ranged from 0.91 to 0.96 for glucose, TG, and total, HDL and LDL cholesterol). ${ }^{22}$

\section{Statistical analysis}

The differences in the baseline characteristics of the mothers and the neonates were evaluated using an analysis of variance and Tukey's honestly significant difference test or the Kruskal-Wallis test and the Dwass-Steele test, depending on the distributions. A comparison of categorical data was carried out using the $\chi^{2}$ test or Fisher's exact test. The medians of individual metabolites were compared between the groups using the Kruskal-Wallis and the Dwass-Steele tests. For each metabolite, the association with BW was assessed by the following: (1) an unadjusted linear regression analysis; (2) a linear regression analysis adjusted for possible confounding factors determined a priori (mode of delivery, maternal prepregnancy Body Mass Index (pBMI), gestational weight gain (GWG), and HbAlc); and (3) if there was a significant association in the previous regression analyses, a possible interaction between metabolite and treatment group was evaluated both with and without additional confounding factors. Mode of delivery acts as a surrogate of fetal stress during the labor, which may affect cord serum metabolites, and was thus included as covariant in the analyses.

Statistical analyses were completed using $\mathrm{R}$ statistical software V.3.6.1 (http://cran.r-project.org). To limit the possibility of type I error, a $p$ value of $<0.01$ was considered statistically significant in the metabolite analyses. Otherwise, for interaction terms and differences in maternal and neonatal characteristics, a $p$ value threshold of 0.05 was employed.

\section{RESULTS}

Population characteristics are shown in table 1. The OGTT 1-hour glucose value was lower in the diet group compared with the metformin group $(10.9 \pm 1.05$ vs $11.3 \pm 1.47 \mathrm{mmol} / \mathrm{L}, \mathrm{p}=0.0061)$, and the incidence of induction of labor was higher in the insulin group $(55.7 \%)$ compared with the diet $(31.9 \%, \mathrm{p}=0.0025)$ or the metformin group $(36.7 \%, \mathrm{p}=0.036)$. Maternal age, HbA1c at the time of diagnosis, and OGTT fasting and 2-hour glucose values tended to be lower in the diet group, although none of these differences were significant according to between-group comparisons. Otherwise, the three groups were similar regarding maternal and neonatal baseline characteristics and clinical outcome data.

Cord serum alanine was significantly higher in the offspring of metformin-treated mothers $(0.53 \mathrm{mmol} / \mathrm{L})$ compared with the insulin $(0.45 \mathrm{mmol} / \mathrm{L}, \mathrm{p}<0.001)$ or the diet groups $(0.46 \mathrm{mmol} / \mathrm{L} ; \mathrm{p}<0.0001$; figure 1$)$. No other metabolite was significantly different between the three groups (online supplemental table 1). Within the metformin group, cord serum metabolites were similar between all pregnancies with or without additional insulin treatment. Moreover, the cord serum alanine remained higher in the metformin group compared with other groups even after excluding the pregnancies treated with additional insulin (online supplemental table 2).

The associations between cord serum metabolites and BW were first evaluated using unadjusted regression analyses (figure 2). Very low-density lipoprotein (VLDL) particle size was inversely related to $\mathrm{BW}(-0.26$; $95 \%$ CI -0.37 to $-0.15 \mathrm{SD} / \mathrm{SD}, \mathrm{p}<0.0001$ ), as was the ratio of TG to phosphoglycerides $(-0.24 ; 95 \% \mathrm{CI}-0.36$ to $-0.13 \mathrm{SD} /$ $\mathrm{SD}, \mathrm{p}<0.0001)$. Omega-3 fatty acid (FA) levels, omega-3to-total-FA ratio, and the docosahexaenoic acid (DHA) and monounsaturated fatty acid (MUFA)-to-total-FA ratio were inversely related to BW. The omega-6-to-total-FA ratio, the omega-6-to-omega-3-FA ratio and the MUFAto-polyunsaturated-fatty aid (PUFA) ratio were positively associated with BW.

After adjusting for pBMI, GWG, maternal HbAlc, and the mode of delivery cholesterol level in very large and large HDL, total lipids in very large HDL, and average HDL particle size were significantly associated with BW (figure 2). The ratio of PUFA to total FA was positively and the degree of unsaturation inversely associated with BW when adjusted for the confounding factors.

Histidine was the only amino acid that was significantly and positively associated with BW. Of the glucose metabolism-related metabolites, only 3-hydroxybutyrate and acetone were found to be positively associated with BW.

The association between the omega-6-to-omega-3-FA ratio and $\mathrm{BW}$ was the only significant association affected by maternal treatment (interaction term $\mathrm{p}<0.05$ ). This positive association was significant in the insulin group $(0.34 ; 95 \%$ CI $0.12,0.56 \mathrm{SD} / \mathrm{SD}, \mathrm{p}=0.002)$ but not in the metformin $(0.22 ; 95 \%$ CI -0.010 to $0.45 \mathrm{SD} / \mathrm{SD}, \mathrm{p}=0.06)$ or the diet group $(0.045 ; 95 \% \mathrm{CI}-0.12$ to $0.21 \mathrm{SD} / \mathrm{SD}$, $\mathrm{p}=0.59$ ). After adjusting for confounding factors, the interaction terms were no longer significant.

\section{DISCUSSION}

In the present study, we found that compared with insulin or diet treatment, metformin caused a similar rise in cord serum alanine as seen in the serum of women with $\mathrm{GDM}^{5}$ and in non-pregnant subjects compared with placebo. ${ }^{23}$ Furthermore, a large VLDL particle size, a high TG-tophosphoglycerides ratio, elevated MUFA-to-total-FA ratio, increased DHA level, and increased omega-3 FA level were associated with a smaller BW, whereas the proportion of omega- 6 FA of the total FA was associated with a higher BW.

Elevated cord blood alanine has been found in the presence of GDM. ${ }^{24}{ }^{25}$ However, to our knowledge, the effects of metformin treatment on neonatal serum alanine have not been studied previously. In line with previous findings on the maternal serum amino acid profile ${ }^{5}$ and in non-pregnant individuals, ${ }^{23}{ }^{26}$ the cord serum alanine concentration was significantly higher in the metformin 
Table 1 Study population characteristics

\begin{tabular}{|c|c|c|c|c|c|c|c|}
\hline Variable & Diet & $\mathbf{n}$ & Insulin & $\mathbf{n}$ & Metformin & $\mathbf{n}$ & $P$ value \\
\hline \multicolumn{8}{|l|}{ Maternal characteristics } \\
\hline Age (years) & $30.3 \pm 5.2$ & 113 & $31.8 \pm 5.5$ & 97 & $32.1 \pm 5.1$ & 98 & $0.026 \mathrm{a}$ \\
\hline Smoking & $11(9.82)$ & 112 & $16(16.7)$ & 96 & $9(9.57)$ & 94 & 0.25 \\
\hline Primipara & $52(46)$ & 113 & $45(46.4)$ & 97 & $37(37.8)$ & 98 & 0.38 \\
\hline Prepregnancy BMI $\left(\mathrm{kg} / \mathrm{m}^{2}\right)$ & $29.1 \pm 5.5$ & 110 & $28.8 \pm 4.7$ & 97 & $29.6 \pm 6.1$ & 98 & 0.59 \\
\hline $\mathrm{HbA1c}$ at GDM diagnosis (\%) & $5.38 \pm 0.33$ & 113 & $5.50 \pm 0.35$ & 97 & $5.48 \pm 0.34$ & 98 & $0.02 b$ \\
\hline OGTT fasting glucose ( $\mathrm{mmol} / \mathrm{L})$ & $5.38 \pm 0.42$ & 113 & $5.54 \pm 0.42$ & 97 & $5.52 \pm 0.55$ & 98 & $0.029 b$ \\
\hline OGTT 1-hour glucose (mmol/L) & $10.9 \pm 1.0$ & 113 & $11.2 \pm 1.2$ & 97 & $11.3 \pm 1.5$ & 98 & 0.0099 aa \\
\hline OGTT 2-hour glucose (mmol/L) & $7.89 \pm 1.87$ & 113 & $7.84 \pm 1.74$ & 96 & $8.32 \pm 1.78$ & 97 & 0.042 \\
\hline $\begin{array}{l}\text { Fasting C-peptide at GDM } \\
\text { diagnosis (nmol/L) }\end{array}$ & $1.01 \pm 0.31$ & 95 & $1.05 \pm 0.30$ & 91 & $1.06 \pm 0.33$ & 93 & 0.41 \\
\hline \multicolumn{8}{|l|}{ Pregnancy outcomes } \\
\hline Gestational hypertension & $4(3.54)$ & 113 & $3(3.09)$ & 97 & $2(2.04)$ & 98 & 0.84 \\
\hline Pre-eclampsia & $4(3.54)$ & 113 & $9(9.28)$ & 97 & $5(5.1)$ & 98 & 0.22 \\
\hline Operative vaginal delivery & $7(6.19)$ & 113 & $7(7.22)$ & 97 & $9(9.18)$ & 98 & 0.71 \\
\hline Cesarean delivery & $17(15)$ & 113 & $15(15.5)$ & 97 & $14(14.3)$ & 98 & 0.97 \\
\hline Induction of labor & $36(31.9)$ & 113 & $54(55.7)$ & 97 & $36(36.7)$ & 98 & $0.0013 \mathrm{bbb}, \mathrm{c}$ \\
\hline Gestational weight gain $(\mathrm{kg})$ & $8.6 \pm 5.1$ & 111 & $7.8 \pm 5.3$ & 97 & $8.0 \pm 5.3$ & 98 & 0.47 \\
\hline $\begin{array}{l}\text { Gestational age at delivery } \\
\text { (weeks) }\end{array}$ & $39.3 \pm 2.2$ & 113 & $39.3 \pm 1.6$ & 97 & $39.2 \pm 1.4$ & 98 & 0.83 \\
\hline \multicolumn{8}{|l|}{ Neonatal outcomes } \\
\hline BW (g) & $3530 \pm 550$ & 113 & $3560 \pm 440$ & 97 & $3580 \pm 500$ & 98 & 0.78 \\
\hline BW (SD) & $-0.08 \pm 1.06$ & 113 & $0.12 \pm 0.93$ & 97 & $0.14 \pm 1.08$ & 95 & 0.2 \\
\hline BW (centiles) & $0.47 \pm 0.29$ & 113 & $0.54 \pm 0.29$ & 97 & $0.54 \pm 0.29$ & 95 & 0.18 \\
\hline SGA & $15(13.3)$ & 113 & $8(8.25)$ & 97 & $12(12.6)$ & 95 & 0.46 \\
\hline LGA & $12(10.6)$ & 113 & $15(15.5)$ & 97 & $14(14.7)$ & 95 & 0.53 \\
\hline Admission to NICU & $33(29.2)$ & 113 & $34(35.1)$ & 97 & $30(30.6)$ & 98 & 0.64 \\
\hline Neonate intravenous glucose & $26(23.4)$ & 111 & $21(21.9)$ & 96 & $23(23.5)$ & 98 & 0.96 \\
\hline
\end{tabular}

Data are given as mean \pm SD or $\mathrm{n}(\%)$.

$P$ value is given for comparison of the whole population, and significant differences between each pair of groups are denoted with lowercase letters: a, diet-metformin $p<0.05$; aa, diet-metformin $p<0.01$; b: diet-insulin $p<0.05$; bbb: diet-insulin $p<0.001$, c, insulin-metformin $\mathrm{p}<0.05$.

LGA: BW>90th centile; SGA: BW<10th centile.

BMI, Body Mass Index; BW, birth weight; GDM, gestational diabetes; HbA1c, glycated hemoglobin; LGA, large for gestational age; NICU, neonatal intensive care unit; OGTT, oral glucose tolerance test; SGA, small for gestational age.

group compared with the insulin or diet groups in this study. This finding could be the result of altered placental metabolism or transfer, increased placental transfer in the presence of an elevated maternal gradient, or due to changes in fetal metabolism. Research in both sheep and human pregnancies has demonstrated that the rate of direct alanine transfer from maternal to fetal circulation is minimal, compared with the effects of placental metabolism and placental-fetal transfer. ${ }^{27}{ }^{28}$ Fetal alanine concentrations are, however, highly influenced by maternal concentrations. ${ }^{27} 28$ Metformin crosses the placenta, and concentrations similar to maternal serum metformin are present in fetal blood. ${ }^{7}$ Thus, by inhibiting hepatic gluconeogenesis, ${ }^{29}$ metformin could have caused an increase in fetal alanine and other gluconeogenic substrates, that is, lactate, alike seen in maternal serum. ${ }^{5}$ Although gluconeogenesis in the human fetus is not currently well characterized, the lack of differences found in our study in other gluconeogenic substrates (pyruvate, lactate, glycerol, and glutamine) or in cord serum glucose reduces the likelihood of major alterations in fetal metabolism in this population due to maternal GDM treatment. Therefore, it seems more plausible that the elevated alanine level in the cord serum was due to increased placental release of alanine rather than altered fetal alanine metabolism.

Metformin treatment of GDM has been associated with a higher weight of the offspring during childhood. ${ }^{9}$ 


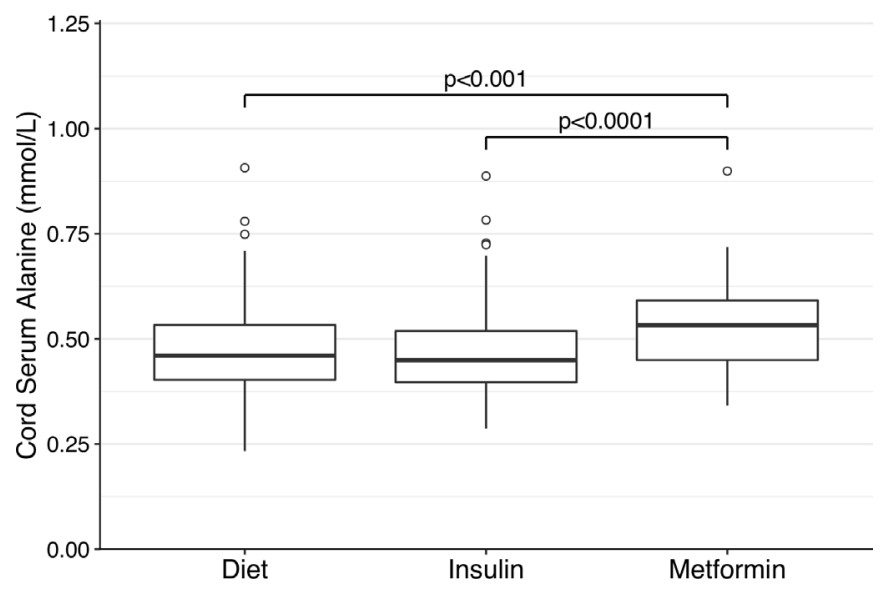

Figure 1 Cord serum alanine concentrations in the diettreated, insulin-treated, and metformin-treated groups. Boxplot representation of the distributions of cord serum alanine concentrations in the diet-treated, insulin-treated and metformin-treated groups, respectively. IQRs with medians are marked with boxes, and the whiskers denote the range. Outliers, which were defined as being more than 1.5 times the IQR away from the median, are marked with circles. P values are given for differences in the Dwass-Steele test and only significant values are shown.

In a birth cohort study, cord serum alanine was positively related to postnatal weight gain and BMI at 2 and 15 years of age, but the association was not significant after Bonferroni correction. ${ }^{14}$ Whether increased fetal serum alanine during late pregnancy could be related to an increased risk of weight gain later in postnatal life warrants further studies.

The degree of maternal glycemia is strongly related with $\mathrm{BW},{ }^{1}$ and measures of maternal glycemia (HbAlc) and endogenous insulin production (C-peptide) have previously been shown to be correlated with both alanine $^{5}$ and $\mathrm{BW}^{10}$ Although alanine concentration in the maternal serum was related to $\mathrm{BW},{ }^{5}$ in the present study, alanine concentration in the cord serum was not. Therefore, higher maternal alanine levels may reflect maternal insulin resistance, and insulin resistance seems to be the common denominator explaining the association between maternal alanine and BW. Consequently, the ability of alanine to accelerate fetal growth directly seems less plausible.

In fetal sheep, the infusion of alanine resulted in elevations of glucose and insulin secretion. ${ }^{30}$ The achieved alanine concentrations were $0.50-0.75 \mathrm{mmol} / \mathrm{L}$ higher compared with baseline. In our study, the cord serum alanine level was $<0.1 \mathrm{mmol} / \mathrm{L}$ higher in the metformin group; therefore, the results of these two studies are not well comparable. Moreover, the cord serum glucose was not higher in neonates exposed to metformin compared with the insulin and diet groups. Previously in a birth cohort study, the cord vein alanine level had an inverse association with BW, although this finding was not significant after Bonferroni correction. ${ }^{14}$ In contrast, in SGA neonates, the cord alanine was decreased; ${ }^{31}$ in another large study, several cord blood amino acids, but not alanine, were related to $\mathrm{BW}^{32}$

Metformin treatment in GDM leads to higher maternal concentrations of total TG, ${ }^{40}$ VLDL TG, VLDL cholesterol, MUFA, SFA, and total FA. ${ }^{4}$ However, these changes were not reflected in the cord serum lipid levels.

It has been proposed that in the context of GDM, cord blood TG levels are inversely related to BW, due to a greater amount of adipose tissue in large fetuses and thus enhanced adipose tissue FA uptake from circulating TG. ${ }^{12}$ This uptake may be further promoted by fetal hyperinsulinemia in pregnancies complicated by hyperglycemia. ${ }^{1}$ Accordingly, in growth-restricted fetuses with presumably less adipose tissue, the cord plasma TG level is elevated. ${ }^{33}$ In addition, we also observed an inverse relationship between BW, VLDL particle size, and the TG-tophosphoglycerides ratio, which both reflect the amount of TG in lipoproteins. In previous studies, the cord vein serum VLDL particle concentration and VLDL TG were elevated in growth-restricted ${ }^{33} 34$ and SGA $^{35}$ neonates, whereas there was no difference in HDL TG level. ${ }^{34}$ As the inverse association with $\mathrm{BW}$ was most evident in the VLDL lipids that supply peripheral tissues, our results support the hypothesis of increased FA uptake from circulating TG in large fetuses.

While the concentrations of LDL and VLDL in cord serum are low, the HDL concentration is similar to nonpregnant adult serum. The apolipoprotein composition of fetal HDL, however, differs from that of adult serum with a relative abundance of apoE. ${ }^{36}$ ApoE serves as a ligand for a variety of receptors (including LDL receptors) and therefore the greater ability of fetal HDL to transport cholesterol between fetal tissues. Cord serum HDL cholesterol has been shown to be related to insulinlike growth factor- 1 and $\mathrm{BW}^{35}$ and is decreased in SGA neonates, ${ }^{37}$ although some studies have found no difference between SGA and appropriately grown neonates. ${ }^{34}$ Thus, it was not unexpected to find a positive association between cord serum cholesterol in very large and large HDL and BW. This association was significant after adjustment for maternal pBMI, GWG, and HbA1c, which suggests that fetal HDL may play a role in fetal growth that is partially independent of maternal characteristics and glycemic control. Moreover, the association was still identified independently of the treatment group.

The importance of PUFA in fetal development has been previously characterized. Long-chain PUFAs, in particular, are required by the central nervous system and for retinal development; during the third trimester, the placenta has selectivity towards the transfer of these lipids. In contrast, maternal omega- 6 FA levels have been positively related to infant obesity. ${ }^{38}$ Similarly, a high omega-6-to-omega-3 ratio in the maternal diet, maternal plasma, and cord plasma are associated with an increased risk of infant obesity at 3 years of age ${ }^{39}$ Arachidonic acid, an omega-6 PUFA, may promote differentiation from preadipocytes into adipocytes, ${ }^{40}$ while DHA inhibits this differentiation. ${ }^{41}$ Accordingly, we found that high 


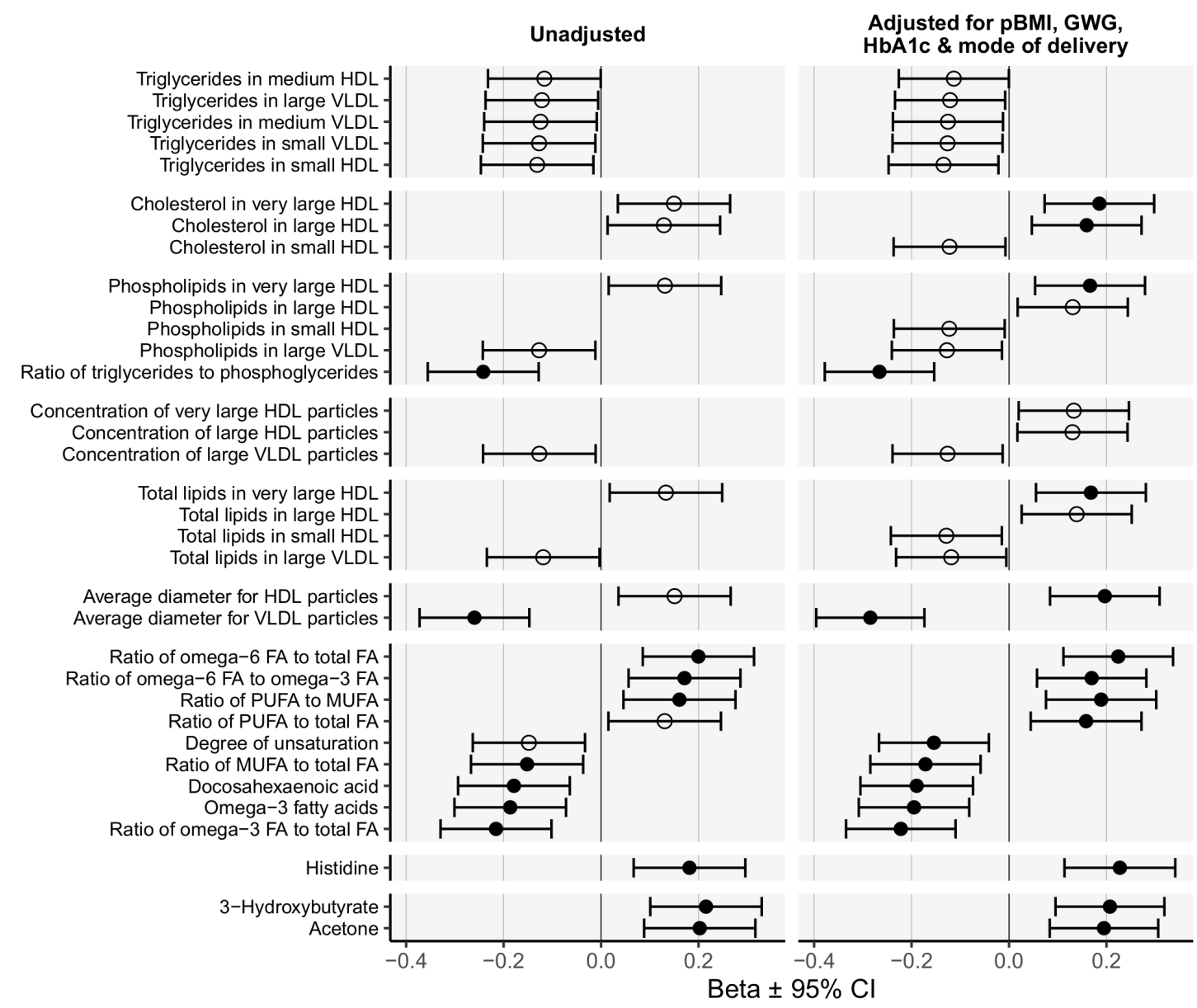

Figure 2 Summary of significant associations between cord serum metabolites and adjusted birth weight. Associations between cord serum metabolites and birth weight in the metformin-treated, insulin-treated, and diet-treated groups combined. Beta values (SD/SD) with 95\% Cls are shown for unadjusted linear regressions, and regression models adjusted for maternal BMI, GWG, maternal HbA1c, and mode of delivery. BW was expressed in SD units (ie, deviation from the Finnish population's mean BW adjusted for gestation duration). Open circles denote $p<0.05$, and closed circles indicate $p<0.01$. Only associations with $p<0.05$ are shown. BW, birth weight; FA, fatty acid; GWG, gestational weight gain; HbA1c, glycated hemoglobin; HDL, high-density lipoprotein; MUFA, monounsaturated fatty acid; pBMI, prepregnancy Body Mass Index; PUFA, polyunsaturated fatty acid; VLDL, very low-density lipoprotein.

omega-6-to-omega-3-FA, and omega-6-to-total-FA ratios as well as high PUFA-to-MUFA, and high PUFA-to-total-FA ratios were positively related to BW. Conversely, a high cord serum degree of FA unsaturation and high concentrations of omega-3 FA (including DHA and high omega3-FA-to-total-FA, and MUFA-to-total-FA ratios) were inversely associated with BW.

Previously, cord blood DHA has been inversely ${ }^{42} 43$ and arachidonic acid has been positively ${ }^{44}$ related to BW, although in some studies, arachidonic acid was also inversely related to $\mathrm{BW} .^{43}$ The divergent results in these two studies and our study may reflect differences in methodology but also the fact that our cord blood samples obtained from neonates exposed to GDM are not representative of the general maternal population. In pregnancies complicated by GDM reduced umbilical arterial omega-3 FA, omega-6 FA, and PUFA levels have been found, suggesting an altered fetal metabolism of these lipids. ${ }^{45}$
The proportion of serum omega- 3 and omega- 6 FA of total FA decreased in both metformin-treated and insulin-treated patients with GDM during the last half of pregnancy. ${ }^{4}$ However, compared with insulin, metformin led to a greater decrease in the proportion of omega- 6 FA and PUFA to total FA. Controversially, omega-6 FA concentration in the maternal serum was associated with BW only in metformin-treated patients, ${ }^{4}$ whereas the association between the cord serum omega-6-to-omega3-FA ratio and BW was significant only in the insulintreated group. Because there was no interaction between the treatment group and other associations of BW with cord serum PUFA and omega FA and the regression beta values with $95 \%$ CIs were rather similar between the insulin-treated (0.34; 95\% CI 0.12 to $0.56 \mathrm{SD} / \mathrm{SD})$ and metformin-treated groups $(0.22 ; 95 \%$ CI -0.010 to 0.45 $\mathrm{SD} / \mathrm{SD})$, this could be an incidental finding. Moreover, the treatment $\times$ metabolite interaction was no longer significant after adjusting for confounding factors. 
Our results suggest that a high cord serum omega-6to-omega-3-FA ratio, which has previously been associated with cardiovascular risk, ${ }^{46}$ is positively associated with BW in the offspring of mothers with GDM. Whether increased fetal adiposity causes increased uptake of MUFA and omega- 6 FA or whether the increased omega6-to-omega-3-FA ratio promotes fetal adipogenesis is not known. Based on previous studies, maternal serum concentrations explain only a small amount of variation in cord serum PUFA, ${ }^{47}$ and although maternal PUFA and omega-6 FA were positively related with BW in metformintreated patients with GDM, ${ }^{4}$ these associations between cord lipids and BW are not affected by maternal treatment. Because similar associations have been found between maternal diet and the plasma omega-6-to-omega3-FA ratio and measures of infant obesity, ${ }^{39}$ future trials of GDM treatment should perhaps focus more specifically on dietary fats.

Histidine was the only amino acid associated with BW. In a previous study, histidine, glycine, and taurine were downregulated in SGA fetuses compared with appropriately growing controls. ${ }^{48}$ In a different population cohort, histidine was positively related to BW. ${ }^{14}$ The fact that only cord serum histidine showed an association with BW in our study seems paradoxical since the placental transfer of several other amino acids is also disturbed in GDM. ${ }^{24}$

Although maternal serum lactate is more elevated in metformin-treated compared with insulin-treated patients with GDM, ${ }^{5}$ there was no difference in cord serum lactate levels. In addition, there was no difference between treatment groups in cord serum ketone levels. We are not aware of any other studies that have reported the effects of intrauterine metformin exposure on cord serum lactate or ketone levels.

In our study population of pregnancies with GDM and good glycemic control, cord serum glucose or lactate levels were not significantly associated with BW. Two ketones (3-hydroxybutyrate and acetone) were positively related with BW. Similarly, Lowe $e$ t $a l^{15}$ suggested a link between cord plasma 3-hydroxybutyrate levels and increased BW and fetal adiposity. Since the fetal liver does not produce a significant amount of ketones but 3-hydroxybutyrate crosses the placenta, the ketones present in cord blood are likely of maternal origin. ${ }^{49}$ In the fetus, ketones may be used for oxidation or lipogenesis, but to which extent they are able to independently promote fetal growth is not known.

Despite the efficacy of metformin treatment in GDM, ${ }^{6}$ prenatal metformin exposure due to placental transfer ${ }^{7}$ has raised some concerns over the possible long-term effects of this treatment. It was thus reassuring to find that the cord serum lipid profile in the metformin group did not significantly differ from either the insulin-treated or the diet-treated group. Moreover, there were no differences in cord serum lactate or ketone levels, suggesting that metformin treatment does not compromise fetal glucose metabolism.
Future studies should focus on whether elevated alanine in the offspring of metformin treated mothers continues during the neonatal period and whether cord serum alanine is associated with long-term metabolic traits. Our study was unable to differentiate whether changes in cord serum alanine were caused by alterations in maternal or fetal metabolism. Future research including simultaneous maternal and fetal serum samples may be better equipped to answer this question.

\section{Strengths and limitations of the study}

Our study population represents patients with GDM of mostly Caucasian origin with good glycemic control. The homogeneity of the population simplified the interpretation of the results but also diminished the results' generalizability to other GDM populations. Although the associations between cord serum metabolites and BW were essentially similar between the treatment groups, these associations may be different in pregnancies not complicated by GDM. ${ }^{50}$ We used mixed cord blood samples instead of arterial or venous samples. Although the differences in amino acid, FA, and cholesterol concentrations between umbilical arterial and venous samples are mostly small, ${ }^{24} 4551$ the proportions of arterial and venous blood may have varied to some degree in individual mixed cord blood samples, thus being a potential source of variation in metabolite concentrations. The sampling method, however, was similar across the study groups and therefore should not have caused bias in the results.

\section{CONCLUSIONS}

Metformin treatment in GDM leads to an increase in cord serum alanine but does not affect neonate lipid profile, although metformin has previously been shown to increase maternal TG. ${ }^{410}$ Possible long-term effects of increased cord serum alanine in the offspring require further follow-up studies. Our results support the hypothesis of increased FA uptake from circulating TG in larger fetuses. Cord serum cholesterol in very large HDL, the proportions of PUFA and MUFA of all FA, the PUFA-toMUFA ratio, and the omega-6-to-omega-3-FA ratio are associated with the regulation of fetal growth in GDM. Moreover, these associations seem to be independent of maternal pBMI, GWG, HbAlc, and type of GDM treatment.

Acknowledgements We thank all the pregnant women who participated in the study and all midwives and physicians at Turku University Hospital who participated in the original study and contributed to the collection of the serum samples.

Contributors KT provided clinical data on the metformin-treated and insulintreated patients and the cord serum samples of all patients from a previous study. OP provided clinical data of the diet treated patients and reviewed the manuscript. MSH analyzed the data and wrote the first draft of the manuscript. KT and TR designed the present study and revised the manuscript. All authors approved the final version of the manuscript.

Funding This study was funded by the Turku University Hospital Foundation (Finland), state funding for university-level health research (Finland), the Diabetes Research Foundation (Finland), and the Finnish Medical Foundation (Finland). 
Competing interests None declared.

\section{Patient consent for publication Not required.}

Ethics approval The original randomized trial was approved by the ethics committee of the Southwest Hospital District of Finland (Dnro 246/2005), the Finnish National Agency of Medicines, and the European Union Drug Regulatory Agency (EUDRA, EUDRA Ct number 2005-004048-30). All participants provided written informed consent.

Provenance and peer review Not commissioned; externally peer reviewed. Data availability statement The data used and/or analyzed during the current study are available from the corresponding author on a reasonable request.

Supplemental material This content has been supplied by the author(s). It has not been vetted by BMJ Publishing Group Limited (BMJ) and may not have been peer-reviewed. Any opinions or recommendations discussed are solely those of the author(s) and are not endorsed by BMJ. BMJ disclaims all liability and responsibility arising from any reliance placed on the content. Where the content includes any translated material, BMJ does not warrant the accuracy and reliability of the translations (including but not limited to local regulations, clinical guidelines, terminology, drug names and drug dosages), and is not responsible for any error and/or omissions arising from translation and adaptation or otherwise.

Open access This is an open access article distributed in accordance with the Creative Commons Attribution Non Commercial (CC BY-NC 4.0) license, which permits others to distribute, remix, adapt, build upon this work non-commercially, and license their derivative works on different terms, provided the original work is properly cited, appropriate credit is given, any changes made indicated, and the use is non-commercial. See: http://creativecommons.org/licenses/by-nc/4.0/.

ORCID iD

Mikael S Huhtala http://orcid.org/0000-0001-5564-9788

\section{REFERENCES}

1 HAPO Study Cooperative Research Group, Metzger BE, Lowe LP, et al. Hyperglycemia and adverse pregnancy outcomes. N Engl J Med 2008;358:1991-2002.

2 Ben-Haroush A, Yogev Y, Hod M. Epidemiology of gestational diabetes mellitus and its association with type 2 diabetes. Diabet Med 2004;21:103-13.

3 Clausen TD, Mathiesen ER, Hansen T, et al. Overweight and the metabolic syndrome in adult offspring of women with diet-treated gestational diabetes mellitus or type 1 diabetes. $J$ Clin Endocrinol Metab 2009;94:2464-70.

4 Huhtala MS, Tertti K, Rönnemaa T. Serum lipids and their association with birth weight in metformin and insulin treated patients with gestational diabetes. Diabetes Res Clin Pract 2020;170:108456.

5 Huhtala MS, Tertti K, Pellonperä O, et al. Amino acid profile in women with gestational diabetes mellitus treated with metformin or insulin. Diabetes Res Clin Pract 2018;146:8-17.

6 Farrar D, Simmonds M, Bryant M, et al. Treatments for gestational diabetes: a systematic review and meta-analysis. BMJ Open 2017;7:e015557.

7 Tertti K, Ekblad U, Heikkinen T, et al. The role of organic cation transporters (OCTs) in the transfer of metformin in the dually perfused human placenta. Eur J Pharm Sci 2010;39:76-81.

8 van Weelden W, Wekker V, de Wit L, et al. Long-Term effects of oral antidiabetic drugs during pregnancy on offspring: a systematic review and meta-analysis of follow-up studies of RCTs. Diabetes Ther 2018;9:1811-29.

9 Rowan JA, Rush EC, Plank LD, et al. Metformin in gestational diabetes: the offspring follow-up (MiG TOFU): body composition and metabolic outcomes at 7-9 years of age. BMJ Open Diabetes Res Care 2018;6:e000456.

10 Barrett HL, Gatford KL, Houda CM, et al. Maternal and neonatal circulating markers of metabolic and cardiovascular risk in the metformin in gestational diabetes (mig) trial: responses to maternal metformin versus insulin treatment. Diabetes Care 2013;36:529-36.

11 Scholtens DM, Bain JR, Reisetter AC, et al. Metabolic networks and metabolites underlie associations between maternal glucose during pregnancy and newborn size at birth. Diabetes 2016;65:2039-50.

12 Schaefer-Graf UM, Graf K, Kulbacka I, et al. Maternal lipids as strong determinants of fetal environment and growth in pregnancies with gestational diabetes mellitus. Diabetes Care 2008;31:1858-63.

13 Kadakia R, Nodzenski M, Talbot O, et al. Maternal metabolites during pregnancy are associated with newborn outcomes and hyperinsulinaemia across ancestries. Diabetologia 2019;62:473-84.
14 Hellmuth C, Uhl O, Standl M, et al. Cord blood metabolome is highly associated with birth weight, but less predictive for later weight development. Obes Facts 2017;10:85-100.

15 Lowe WL, Bain JR, Nodzenski M, et al. Maternal BMI and glycemia impact the fetal metabolome. Diabetes Care 2017;40:902-10.

16 Rump P, Popp-Snijders C, Heine RJ, et al. Components of the insulin resistance syndrome in seven-year-old children: relations with birth weight and the polyunsaturated fatty acid content of umbilical cord plasma phospholipids. Diabetologia 2002;45:349-55.

17 Shokry E, Marchioro L, Uhl O, et al. Transgenerational cycle of obesity and diabetes: investigating possible metabolic precursors in cord blood from the PREOBE study. Acta Diabetol 2019;56:1073-82.

18 Tertti K, Ekblad U, Koskinen P, et al. Metformin vs. insulin in gestational diabetes. A randomized study characterizing metformin patients needing additional insulin. Diabetes Obes Metab 2013;15:246-51.

19 Pellonperä O, Rönnemaa T, Ekblad U, et al. The effects of metformin treatment of gestational diabetes on maternal weight and glucose tolerance postpartum--a prospective follow-up study. Acta Obstet Gynecol Scand 2016;95:79-87.

20 Pihkala J, Hakala T, Voutilainen P, et al. [Characteristic of recent fetal growth curves in Finland]. Duodecim 1989;105:1540-6 http://www. ncbi.nlm.nih.gov/pubmed/2680445

21 Soininen P, Kangas AJ, Würtz P, et al. Quantitative serum nuclear magnetic resonance metabolomics in cardiovascular epidemiology and genetics. Circ Cardiovasc Genet 2015;8:192-206.

22 Würtz P, Kangas AJ, Soininen P, et al. Quantitative serum nuclear magnetic resonance metabolomics in large-scale epidemiology: a primer on -Omic technologies. Am J Epidemiol 2017;186:1084-96.

23 Preiss D, Rankin N, Welsh P, et al. Effect of metformin therapy on circulating amino acids in a randomized trial: the CAMERA study. Diabet Med 2016;33:1569-74.

24 Cetin I, de Santis MSN, Taricco E, et al. Maternal and fetal amino acid concentrations in normal pregnancies and in pregnancies with gestational diabetes mellitus. Am J Obstet Gynecol 2005;192:610-7.

25 Dani C, Bresci C, Berti E, et al. Metabolomic profile of term infants of gestational diabetic mothers. J Matern Fetal Neonatal Med 2014:27:537-42.

26 Eppinga RN, Kofink D, Dullaart RPF, et al. Effect of metformin on metabolites and relation with myocardial infarct size and left ventricular ejection fraction after myocardial infarction. Circ Cardiovasc Genet 2017;10:e001564.

27 Timmerman M, Chung M, Wilkening RB, et al. Relationship of fetal alanine uptake and placental alanine metabolism to maternal plasma alanine concentration. Am J Physiol 1998;275:E942-50.

28 Holm MB, Bastani NE, Holme AM, et al. Uptake and release of amino acids in the fetal-placental unit in human pregnancies. PLOS One 2017:12:1-15.

29 Hundal RS, Krssak M, Dufour S, et al. Mechanism by which metformin reduces glucose production in type 2 diabetes. Diabetes 2000;49:2063-9.

30 Philipps AF, Dubin JW, Raye JR. Alanine-stimulated insulin secretion in the fetal and neonatal lamb. Am J Obstet Gynecol 1980;136:597-602.

31 Ivorra C, García-Vicent C, Chaves FJ, et al. Metabolomic profiling in blood from umbilical cords of low birth weight newborns. J Trans/ Med 2012;10:142-10.

32 Kadakia R, Talbot O, Kuang A, et al. Cord blood metabolomics: association with newborn Anthropometrics and C-peptide across ancestries. J Clin Endocrinol Metab 2019;104:4459-72.

33 Sanz-Cortés M, Carbajo RJ, Crispi F, et al. Metabolomic profile of umbilical cord blood plasma from early and late intrauterine growth restricted (IUGR) neonates with and without signs of brain vasodilation. PLoS One 2013;8:e80121-21.

34 Miranda J, Simões RV, Paules C, et al. Metabolic profiling and targeted lipidomics reveals a disturbed lipid profile in mothers and fetuses with intrauterine growth restriction. Sci Rep 2018;8:13614.

35 Nagano N, Okada T, Fukamachi R, et al. Insulin-Like growth factor-1 and lipoprotein profile in cord blood of preterm small for gestational age infants. J Dev Orig Health Dis 2013;4:507-12.

36 Nagasaka $\mathrm{H}$, Chiba $\mathrm{H}$, Kikuta $\mathrm{H}$, et al. Unique character and metabolism of high density lipoprotein (HDL) in fetus. Atherosclerosis 2002;161:215-23.

37 Pecks U, Brieger M, Schiessl B, et al. Maternal and fetal cord blood lipids in intrauterine growth restriction. $J$ Perinat Med 2012;40:287-96.

38 Moon RJ, Harvey NC, Robinson SM, et al. Maternal plasma polyunsaturated fatty acid status in late pregnancy is associated with offspring body composition in childhood. J Clin Endocrinol Metab 2013;98:299-307. 
39 Donahue SMA, Rifas-Shiman SL, Gold DR, et al. Prenatal fatty acid status and child adiposity at age 3 y: results from a US pregnancy cohort. Am J Clin Nutr 2011;93:780-8.

40 Gaillard D, Négrel R, Lagarde M, et al. Requirement and role of arachidonic acid in the differentiation of pre-adipose cells. Biochem J 1989;257:389-97.

41 Kim H-K, Della-Fera M, Lin J, et al. Docosahexaenoic acid inhibits adipocyte differentiation and induces apoptosis in 3T3-L1 preadipocytes. J Nutr 2006;136:2965-9.

42 Robinson O, Keski-Rahkonen P, Chatzi L, et al. Cord blood metabolic signatures of birth weight: a population-based study. $J$ Proteome Res 2018;17:1235-47.

43 Rump P, Mensink RP, Kester AD, et al. Essential fatty acid composition of plasma phospholipids and birth weight: a study in term neonates. Am J Clin Nutr 2001;73:797-806.

44 Elias SL, Innis SM. Infant plasma trans, n-6, and n-3 fatty acids and conjugated linoleic acids are related to maternal plasma fatty acids, length of gestation, and birth weight and length. Am J Clin Nutr 2001;73:807-14
45 Ortega-Senovilla H, Alvino G, Taricco E, et al. Gestational diabetes mellitus upsets the proportion of fatty acids in umbilical arterial but not venous plasma. Diabetes Care 2009;32:120-2.

46 Harris WS. The omega-6/omega-3 ratio and cardiovascular disease risk: uses and abuses. Curr Cardiovasc Risk Rep 2007;1:39-45.

47 Hendrickse W, Stammers JP, Hull D. The transfer of free fatty acids across the human placenta. Br J Obstet Gynaecol 1985;92:945-52.

48 Cetin I, Corbetta C, Sereni LP, et al. Umbilical amino acid concentrations in normal and growth-retarded fetuses sampled in utero by cordocentesis. Am J Obstet Gynecol 1990;162:253-61.

49 Herrera E, Amusquivar E, López-Soldado I, et al. Maternal lipid metabolism and placental lipid transfer. Horm Res 2006;65 Suppl 3:59-64.

50 Schaefer-Graf UM, Meitzner K, Ortega-Senovilla H, et al. Differences in the implications of maternal lipids on fetal metabolism and growth between gestational diabetes mellitus and control pregnancies. Diabet Med 2011;28:1053-9.

51 Horne H, Holme AM, Roland MCP, et al. Maternal-Fetal cholesterol transfer in human term pregnancies. Placenta 2019;87:23-9. 